\title{
The Home Independence Program with non- health professionals as care managers: an evaluation
}

This article was published in the following Dove Press journal:

Clinical Interventions in Aging

20 June 2016

Number of times this article has been viewed

\section{Gill Lewin' \\ Karyn Concanen ${ }^{2}$ \\ David Youens ${ }^{3}$}

'School of Nursing, Midwifery and Paramedicine, Curtin University, Perth, WA, Australia; ${ }^{2}$ Silver Chain Group, Osborne Park, WA, Australia; ${ }^{3}$ Faculty of Health Science, Curtin University, Perth, WA, Australia
Correspondence: Gill Lewin School of Nursing, Midwifery and Paramedicine, Curtin University, GPO Box U1987, Perth WA 6845, Australia Email g.lewin@curtin.edu.au
Abstract: The Home Independence Program (HIP), an Australian restorative home care/reablement service for older adults, has been shown to be effective in reducing functional dependency and increasing functional mobility, confidence in everyday activities, and quality of life. These gains were found to translate into a reduced need for ongoing care services and reduced health and aged care costs over time. Despite these positive outcomes, few Australian home care agencies have adopted the service model - a key reason being that few Australian providers employ health professionals, who act as care managers under the HIP service model. A call for proposals from Health Workforce Australia for projects to expand the scope of practice of health/aged care staff then provided the opportunity to develop, implement, and evaluate a service delivery model, in which nonprofessionals replaced the health professionals as Care Managers in the HIP service. Seventy older people who received the HIP Coordinator (HIPC) service participated in the outcomes evaluation. On a range of personal outcome measures, the group showed statistically significant improvement at 3 and 12 months compared to baseline. On each outcome, the improvement observed was larger than that observed in a previous trial in which the service was delivered by health professionals. However, differences in the timing of data collection between the two studies mean that a direct comparison cannot be made. Clients in both studies showed a similarly reduced need for ongoing home care services at both follow-up points. The outcomes achieved by HIPC, with non-health professionals as Care Managers, were positive and can be considered to compare favorably with the outcomes achieved in HIP when health professionals take the Care Manager role. These findings will be of interest to managers of home care services and to policy makers interested in reducing the long-term care needs of older community dwelling individuals.

Keywords: evaluation, home care, models of service delivery, rehabilitation, social care

\section{Introduction}

To offset the increased demand for health and aged care services associated with population aging, governments and service providers around the world have become increasingly interested in interventions that can actively reduce an individual's service needs. Restorative home care or reablement services are designed to do exactly that. These types of services have been found to significantly increase the likelihood that an older person referred for home care will not need ongoing home care assistance, $;^{1-4}$ significantly reduce the likelihood of admission to residential care, ${ }^{5,6}$ hospital, ${ }^{7,8}$ or presentation to an emergency department; $;^{7,9}$ and affect the rate and type of home care support needed for up to 5 years afterwards. ${ }^{10}$ 
Although having many elements in common, reablement and restorative home care services differ between countries and, within countries, between providers. Having identified that these types of interventions "work", it is important that we understand whether there are elements that are critical in determining effectiveness; how these services can be most cost effectively delivered; and whether they are differentially effective depending on the characteristics of the service recipient. To date, there has been little research addressing any of these questions. Although, Cochrane et $\mathrm{al}^{11}$ have, in order to conduct a systematic review of the effectiveness of reablement services, identified five essential defining criteria for an intervention to be called reablement:

1. participants must have an identified need for formal care and support, or are at risk of functional decline; ${ }^{1}$

2. the intervention must be time-limited (typically 6-12 weeks) and intensive (eg, multiple home visits); ${ }^{12}$

3. the intervention must be delivered in the older person's own home; ${ }^{13}$

4. the intervention must focus on maximizing independence; and

5. the intervention must be person-centered and goaldirected. $^{14}$

The Home Independence Program (HIP), an Australian restorative home care service, meets these criteria and has been the subject of a number of studies that have demonstrated its effectiveness both in terms of individual client outcomes and reduction in use of home and health care services. ${ }^{3,7,10,15}$ As originally developed, and operated within all previous research, the HIP service delivery model requires a multidisciplinary team of health professionals (namely occupational therapists [OTs], physiotherapists [physios] and nurses) to work in an interdisciplinary way, so that one team member acts as the Care Manager for each client. This means assessing, goal setting, care-planning, and working with the client to implement any of a core set of interventions/ strategies relevant to the individual's goals, regardless of the professional origin of the intervention/strategy. This way of working is enabled by the following: weekly team meetings in which all new clients are discussed; the ability of team members to consult with each other or refer to another team member as a specialist when something more than, or different from, the core generic intervention is required; and professional development sessions during which team members are coached in the delivery of the core interventions by one or more of the professionals for whom the intervention formed part of their basic training. For example, the physiotherapists lead the training on the strength and balance exercise program, the OTs lead that on task analysis and design, and the nurses lead that on medication management.

Over the years that the HIP service has been operating, it has been difficult for the provider organization to maintain the team delivering the service at full strength due to a shortage of allied health staff with community-based experience. There has also been less take up of the HIP service model by other home care agencies than hoped by both its developers and the West Australian Health Department who encouraged its development and subsequent testing and fund it on an ongoing basis through the Home and Community Care Program (HACC). This was thought to be at least in part due to few Australian home care agencies employing allied health and nursing staff.

In the UK, reablement services operate successfully with many different service models, ${ }^{16}$ many of which use teams entirely of non-health professionals. Although it is considered that occupational therapy skills and knowledge have an important role in the delivery of reablement, it is not thought essential that OTs are core team members if timely access can be assured, and if they are involved in the training of the reablement workers. ${ }^{17}$

Given these three factors, it was decided that it was important to determine whether it was possible to achieve as good service outcomes with a HIP service delivery model, in which non-health professionals were trained to be the Care Managers, as was achieved with health professional Care Managers.

A call for proposals by Health Workforce Australia (HWA) for projects to expand the scope of practice of health/aged care workers then provided the opportunity and impetus to develop a proposal for how this could be done. HWA liked the proposal and provided funding to develop and implement the service model and coordinator training for a HIP Coordinator (HIPC) service. The Western Australian (WA) Department of Health, who supported the submission to HWA, then agreed to fund an evaluation of the new service when implemented. This paper focuses on the outcomes aspect of that evaluation.

\section{Methods}

\section{Study design}

Prior to commencement, this study received approval from the Silver Chain Human Research Ethics Committee. The evaluation was designed to examine both service processes and outcomes - the former to ensure that the HIPC service was implemented as designed, and the latter to assess whether the outcomes achieved by the service were comparable to 
others reported in the literature and, in particular, how they compared to the outcomes achieved in an earlier randomized controlled trial $(\mathrm{RCT})^{2}$ when HIP was delivered by health professionals acting as the Care managers. The outcomes were examined using a quasiexperimental pre-post study design and included both individual and service outcomes.

The process evaluation was completed between March and December 2012, and an internal report summarizing the findings and the associated recommended actions, already provided to the operational team at regular meetings, was provided to service management early in January 2013. It will not therefore be described further here. The outcomes evaluation was conducted between January 2013 and December 2014.

\section{Study setting}

HIPC was implemented across the Perth metropolitan area by the large community health and aged care organization that has been providing HIP in Western Australia for the last 12 years.

\section{Study participants}

The eligibility criteria for receipt of a HIPC service included the following: being referred to a government-funded Regional Assessment Service for home care; assessed as eligible for that service to be funded by HACC; and interested in receiving a service taking a restorative approach. The exclusion criteria included having a diagnosis of dementia or other progressive neurological condition.

Based on expected referral rates and project timelines, the intended sample size was 100; of which 25 were from each of the four Perth metropolitan regions. This sample size was calculated as providing sufficient power $(>80 \%)$ to assess the significance of a moderate difference (14\%) in the proportion of clients with different service outcomes as compared to the HIP RCT. Unfortunately, referrals to the service were initially slower than had been anticipated, and it was necessary to stop recruitment at 70 clients to fit within research timeframes. This reduced sample size was calculated as providing power to assess the significance of a slightly larger difference (16\%) in the proportion of clients with different service outcomes as compared to the HIP RCT.

\section{The HIPC service}

The HIPC service model included all the same key components as the original HIP service model:3,18

- Comprehensive multidimensional assessment;

- Goal-oriented care planning in partnership with client;
- Targeted evidence-based interventions to optimize functioning in daily living activities;

- Minimized face-to-face contact - telephone support and follow-up;

- Education about self-management, healthy aging, use of medications, and illness/accident prevention strategies;

- Use of language and patterns of communication that encourage clients and families to participate in all care decisions and which promote their sense of autonomy rather than exerting power or control over the client;

- Recognition of the importance of the social support aspect of home care services for older people and the need to assist the client to develop other avenues for gaining this support; and

- Use of local resources - facilitated by a resource file.

The types of interventions included in an individual's care plan were based on an individual's goals, their capabilities, and the types of difficulties they were experiencing. They might include task analysis and redesign; work simplification; assistive technology; strength, balance, and endurance programs; chronic disease self-management; fall prevention strategies; and medication, continence, or nutrition management.

The training provided to the coordinators included training on all the key components and specific interventions using a combination of presentations, case studies, role plays, activities, take home exercises, and prereading. It was followed by competency assessment, buddy shifts, and formal supervision. Training was delivered by the Senior Allied Health Professionals working within the multidisciplinary HIP team. As with the original HIP model, new clients were discussed at weekly multidisciplinary case meetings, and processes were also developed for referral to a specific health professional discipline when an individual's needs were assessed as outside the range of a "standard" intervention.

Again as in HIP, individuals participated in HIPC until they achieved their goals or for up to 12 weeks, whichever came first. In a minority of cases, an individual received the service for longer than 12 weeks as they were considered to be progressing well toward achieving their goals and required some continuing support. If at discharge from HIPC individuals still needed assistance from a home care service, this was set up by the coordinator who then passed over coordination of the care to the home care coordinator.

\section{Data collection}

The outcomes data were collected at three time points: initial HIPC visit (baseline), 3 months, and 1 year. After 
receiving details of a new referral, the HIPC contacted the person to set up a time for the initial assessment visit, and, when a date and time were agreed, telephoned or emailed the research assistant to advise them of the client's details and visit. The research assistant then telephoned the person, briefly described the study and its purpose, invited them to participate, and if they agreed, told them they would be combining their visit with that of the HIPC coordinator. This was an important consideration and served a dual purpose of avoiding contamination of the baseline, as the client had not yet been exposed to any intervention, and minimizing intrusion into the client's life by avoiding an extra visit at a time when they may be anxious or overwhelmed. At the visit, when the client had read the information statement and signed the consent form, the research assistant collected the outcomes data prior to the coordinator conducting the assessment to ensure responses were not influenced by knowledge or expectation of the service. The coordinator simply observed and was able to use what she heard and witnessed during the outcomes measurement as part of her assessment. All research assistants were trained to use the outcomes measures, their data collection was observed, and the reliability of their data was checked by the senior research officer in charge of the project, prior to them commencing unsupervised data collection visits.

Five outcomes measures were used. Activities of Daily Living (ADL) and Instrumental Activities of Daily Living (IADL) data were collected using a tool based on the modified Barthel Index ${ }^{19}$ and the Lawton and Brodie Scale ${ }^{20}$ with a modified scoring system designed to increase as the level of assistance required with a task increased. ${ }^{21}$ The other three measures employed were the Assessment of Quality of Life instrument (AQOL), ${ }^{22}$ the Modified Falls Efficacy Scale (MFES), ${ }^{23}$ and the Timed Up and Go (TUG). ${ }^{24}$

Other data included in the outcomes evaluation were collected as part of routine service provision and included the assessment and demographic data mandated to be collected for the National HACC Minimum Data Set, as well as service activity and outcome data collected within the organization's client information system.

\section{Data management and analysis}

All outcome data were entered into a study database following completion of the final follow-up of the last client. The Minimum Data Set and service activity were then added. Once the data had been cleaned, descriptive statistics were used to summarize participant characteristics, service activity, and outcomes. Additionally, summary statistics were produced to compare demographic characteristics between
HIPC and HIP clients. Comparisons were made between the mean scores at baseline, 3-, and 12-month follow-ups, and the mean change between these time points, for the personal outcomes of HIPC clients and clients who received HIP in the RCT. Paired $t$-tests were used for comparisons of HIPC clients across the three time points, and independent samples $t$-tests were used to compare HIPC clients to clients who received HIP in the RCT. Where data were not normally distributed, Wilcoxon signed-ranks tests and Mann-Whitney $U$-tests were used in place of paired and independent samples $t$-tests, respectively. Analyses of all these outcomes were restricted to include only participants who had complete data for all three data collection points on that outcome, ie, TUG scores were compared at baseline only among those clients who also had 3-and 12-month follow-up data. To test the potential impact of this loss to follow-up, those with complete data were compared to noncompleters on demographic characteristics and baseline scores on each of the measures used, using the aforementioned statistical tests.

To examine the relationship between intervention type and outcome, logistic regression models were used. For each outcome at each follow-up point, a binary variable was generated, where a score of 1 indicated an improvement on the baseline score for an individual and a score of 0 indicated either no change or a poorer score than that recorded at baseline. The effect of receiving the HIPC intervention (as opposed to receiving HIP) on this outcome was estimated, with the additional covariates of age (continuous), sex $(0=$ male, $1=$ female $)$, living arrangement $(0=$ with family or others, $1=$ alone), and baseline score on the relevant outcome measure also included in models. The baseline score on each particular outcome measure was included so as to control for any potential bias caused by differences in the timing of baseline measures between the two studies.

Chi-square tests were used to make comparisons between the proportions of HIPC and HIP RCT clients with different service outcomes. To enable an unbiased comparison of these outcomes, data were taken directly from Comcare, the provider organization's client management system, whereas the data used in the RCT analyses and for interim reporting of HIPC results at conferences had been checked against information from other sources, and modified if needed. The additional sources used for checking service data differed between the HIP RCT and the HIPC interim analysis; hence, these data were excluded from the analyses reported here.

Additionally, the original RCT had focused on service outcomes in terms of personal care (PC) services, as that study was restricted to individuals with $\mathrm{PC}$ needs. However, as the HIPC study had broader selection criteria, a more generic set 
of service outcomes was considered appropriate for comparing the two trials and records relating to all services received by a client over the study period (other than those which do not involve home visits) contributed to their service outcome. Other relevant service data such as deceased date and temporary change in care data were also used to identify outcomes as completely as possible. The method used aimed to achieve 1) accuracy, by identifying as closely as possible each client's situation at each follow-up date, 2) internal validity, by applying the same protocol to HIPC and HIP RCT clients, and 3 ) practicality, by defining outcomes based on Comcare extracts rather than manual review of records, considering the numbers of clients in the two studies.

All analyses were conducted using STATA $13,{ }^{25}$ and a $P$-value of $<0.05$ was taken to indicate statistical significance.

\section{Findings}

\section{Clients}

Of 76 clients, 70 consented to participate in completion of the baseline measures. Over the next 12 months, twelve participants were lost to follow-up. The reasons provided are shown in Figure 1. Table 1 shows that the 12 participants lost to follow-up did not differ significantly from the 58 who remained in the study to completion, either on any of the demographic factors available or in terms of their baseline scores on any of the outcome measures.

The average age of participants was 76 years, with a range from 44 to 91 years. Ninety percent were aged 65 years or over. The majority of clients were female and did not have a carer (Table 2). Just over half the clients were born in Australia, and the majority spoke English as a first language. Most were in receipt of an aged or other government pension. HIPC clients were similar on most demographic measures to clients in the HIP RCT, apart from being significantly younger (mean age 76 years compared to 81 years, $P=0.011$ ) and less likely to have a carer (30\% compared to $50 \%$, $P=0.015)$.

All except two of the clients were referred by a metropolitan regional assessment service. One of these two was a selfreferral, the other person was referred from the hospital. Thirty-one of the clients had previously received, or were still receiving, HACC services. Between them they had had 59 service episodes, the most common (20/59) being Domestic Assistance, but Nursing ran a close second (19/59).

\section{Personal outcomes}

The outcome measure group mean scores at each of the study measurement points are shown in Table 3.

Comparison of the scores for the HIPC clients at the first two time points found a statistically significant improvement on all measures between baseline and 3 months (ADL change $t=5.56, d f$ [degrees of freedom] $=57, P<0.0001$; IADL change $t=8.57, d f=57, P<0.0001$; AQOL change $t=6.11$, $d f=57, P<0.0001$; MFES change $t=-5.12, d f=55, P<0.0001$; TUG change $z=5.36, P<0.00001)$.

Significant improvement on all outcomes was also found between baseline and 12 months, indicating that the

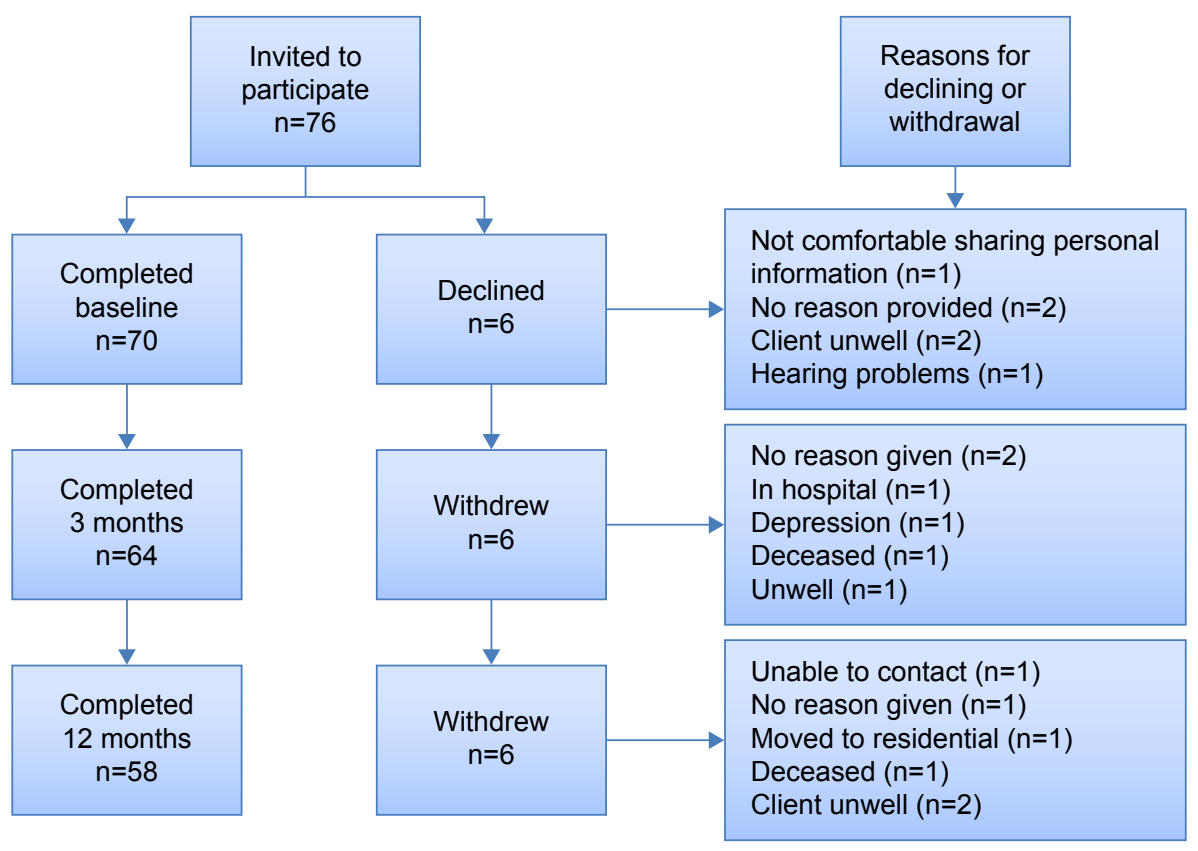

Figure I Participant flow and reasons for loss to follow-up. 
Table I Baseline differences between HIPC completers and noncompleters

\begin{tabular}{llll}
\hline Baseline measure & Completers (n=58) & Noncompleters $(\mathbf{n}=\mathbf{I 2})$ & $P$-values \\
\hline Age (years), mean (SD) & $76.84(9.19)$ & $74(10.60)$ & $0.468^{\mathrm{a}}$ \\
Number of females, $\mathrm{n}(\%)$ & $48(82.76 \%)$ & $10(83.33 \%)$ & $0.664^{\mathrm{b}}$ \\
Number of clients who live alone, $\mathrm{n}(\%)$ & $33(56.90 \%)$ & $5(41.67 \%)$ & $0.259^{\mathrm{b}}$ \\
Has a carer, $\mathrm{n}(\%)$ & $6(50.00 \%)$ & $15(26.32 \%)$ & $0.103^{\mathrm{b}}$ \\
IADL, mean (SD) & $18.84(4.36)$ & $19.42(4.48)$ & $0.766^{\mathrm{a}}$ \\
ADL, mean (SD) & $13.17(2.98)$ & $14.42(4.58)$ & $0.453^{\mathrm{a}}$ \\
TUG, mean (SD) & $26.37(11.98)$ & $22.87(12.20)$ & $0.233^{\mathrm{a}}$ \\
AQOL, mean (SD) & $19.71(5.55)$ & $22.50(6.20)$ & $0.125^{\mathrm{a}}$ \\
MFES, mean (SD) & $6.83(2.01)$ & $7.19(1.57)$ & $0.629^{\mathrm{a}}$ \\
\hline
\end{tabular}

Notes: ${ }^{2}$-value derived using Mann-Whitney U-test; ${ }^{\text {}} P$-value derived using Fisher's exact test.

Abbreviations: HIPC, Home Independence Program Coordinator; SD, standard deviation; ADL, Activities of Daily Living; IADL, Instrumental Activities of Daily Living; AQOL, Assessment of Quality of Life; MFES, Modified Falls Efficacy Scale; TUG, Timed Up and Go.

improvement in functioning was sustained (ADL change $z=3.62, P=0.0003$; IADL change $t=5.44, d f=57, P<0.0001$; AQOL change $t=7.03, d f=57, P<0.0001$; MFES change $t=-5.56, d f=55, P<0.0001$; TUG change $t=7.51, d f=43$, $P<0.0001)$.

However, comparison of scores between 3 and 12 months follow-up found a statistically significant improvement in
TUG score ( $z=1.97, P=0.0486)$, a statistically significant decline in IADLs $(z=-2.05, P=0.0404)$, and no significant change between the two follow-up points for the remaining three outcomes - ADL, MFES, and AQOL. Although IADL scores declined from 3 to 12 months, scores at the 12-month follow-up were still significantly better than at baseline $(t=5.44, d f=57, P<0.0001)$.

Table 2 HIPC client minimum data set demographics compared to HIP RCT clients

\begin{tabular}{|c|c|c|c|}
\hline Client demographic & HIP RCT, n (\%) & HIPC, n (\%) & $P$-value \\
\hline \multicolumn{4}{|l|}{ Sex } \\
\hline Female & 63 (7I.59) & $58(82.86)$ & \multirow[t]{3}{*}{$0.130^{\mathrm{b}}$} \\
\hline Male & $25(28.4 I)$ & $12(17.14)$ & \\
\hline Total & 88 & 70 & \\
\hline \multicolumn{4}{|l|}{ Living arrangements } \\
\hline Lives alone & $52(59.09)$ & $38(54.29)$ & \multirow[t]{3}{*}{$0.628^{\mathrm{b}}$} \\
\hline Lives with family/others & $36(40.91)$ & $32(45.7 I)$ & \\
\hline Total & 88 & 70 & \\
\hline \multicolumn{4}{|l|}{ Carer availability } \\
\hline Has a carer & $44(50.00)$ & $21(30.00)$ & \multirow[t]{4}{*}{$0.015^{b, c}$} \\
\hline Has no carer & $44(50.00)$ & $48(68.57)$ & \\
\hline Not stated & $0(0.00)$ & $\mathrm{I}(\mathrm{I} .43)$ & \\
\hline Total & 88 & 70 & \\
\hline \multicolumn{4}{|l|}{ Country of birth } \\
\hline Australia & $54(6 \mid .36)$ & $38(54.29)$ & \multirow[t]{5}{*}{$0.418^{\mathrm{b}, \mathrm{d}}$} \\
\hline England & $15(17.05)$ & $14(20.00)$ & \\
\hline Italy & I (I.I4) & $3(4.29)$ & \\
\hline Other & $18(20.45)$ & $15(2 \mid .43)$ & \\
\hline Total & 88 & 70 & \\
\hline \multicolumn{4}{|l|}{ Language } \\
\hline English & $87(98.86)$ & $66(94.29)$ & \multirow[t]{3}{*}{$0.171^{\mathrm{b}}$} \\
\hline Non-English & $\mathrm{I}(\mathrm{I} .14)$ & $4(5.7 I)$ & \\
\hline Total & 88 & 70 & \\
\hline \multicolumn{4}{|l|}{ Pension } \\
\hline Aged pension & $66(75.00)$ & $5 \mathrm{I}(72.86)$ & \multirow[t]{4}{*}{$0.092^{\mathrm{b}, \mathrm{e}}$} \\
\hline No government pension & $14(15.91)$ & $5(5.68)$ & \\
\hline Other government pension & $8(9.09)$ & $14(20.00)$ & \\
\hline Total & 88 & 70 & \\
\hline Age (years), mean (SD) & $80.76(0.75)$ & $76.36(1.13)$ & $0.003^{\mathrm{a}}$ \\
\hline
\end{tabular}

Notes: aP-value derived using Mann-Whitney U-test; ${ }^{b}$-value derived using Fisher's exact test. 'One HIPC client with answer "N/S" excluded from significance test; ‘ ${ }^{\circ}$ isher's exact test based on Australia/other; 'Fisher's exact based on pension/no pension.

Abbreviations: HIPC, Home Independence Program Coordinator; HIP RCT, Home Independence Program randomized controlled trial; N/S, not stated; SD, standard deviation. 
Table 3 Outcomes measure mean (SD) scores for HIPC and HIP RCT clients at each point of measurement

\begin{tabular}{|c|c|c|c|c|c|c|}
\hline \multirow[t]{2}{*}{ Measure } & \multicolumn{3}{|l|}{ HIPC } & \multicolumn{3}{|l|}{ HIP RCT } \\
\hline & Baseline & 3 months & I year & Baseline & 3 months & I year \\
\hline$A D L^{a}$ total & 13.17 (2.98) & $11.53(2.17)$ & $11.95(2.72)$ & $12.35(1.88)$ & $11.84(2.06)$ & I2.05 (2.5I) \\
\hline IADL ${ }^{b}$ total & I $8.84(4.36)$ & I3.84 (5.02) & $14.88(6.01)$ & 17.25 (3.77) & I5.56 (4.15) & $15.40(4.60)$ \\
\hline AQOL'c score & |9.7| (5.55) & I5.84 (5.87) & $15.40(6.00)$ & $17.16(5.84)$ & $15.62(6.55)$ & 15.79 (5.62) \\
\hline MFES ${ }^{d}$ score & $6.83(2.03)$ & $8.22(1.63)$ & $8.25(\mathrm{I} .5 \mathrm{I})$ & $7.70(1.82)$ & $7.97(1.70)$ & $7.93(1.92)$ \\
\hline TUGe time in seconds & $25.75(12.30)$ & I6.86 (8.76) & $|5.7|(8.13)$ & 27.49 (18.69) & $23.57(15.66)$ & 24.41 (19.62) \\
\hline
\end{tabular}

Notes: aPAF Activities of Daily Living score of $9=$ independent on all tasks and $29=$ totally dependent on others for all tasks; ${ }^{\mathrm{b} A F}$ Instrumental Activities of Daily Living score of $8=$ independent on all tasks and $30=$ totally dependent on others for all tasks; 'Assessment of Quality of Life, the lower the score the better the quality of life; ${ }^{\text {Modified }}$ Falls Efficacy Scale, the higher the score the more confident the person; ${ }^{\mathrm{e} T i m e d ~ U p ~ a n d ~ G o, ~ t h e ~ g r e a t e r ~ t h e ~ t i m e ~ t a k e n ~ t h e ~ p o o r e r ~ t h e ~ m o b i l i t y . ~}$

Abbreviations: SD, standard deviation; HIPC, Home Independence Program Coordinator; HIP RCT, Home Independence Program randomized controlled trial; PAF, primary assessment form; ADL, Activities of Daily Living; IADL, Instrumental Activities of Daily Living; AQOL, Assessment of Quality of Life; MFES, Modified Falls Efficacy Scale; TUG, Timed Up and Go.

When these results from the HIPC clients were then compared with the results from clients in the earlier HIP RCT (Tables 3 and 4), it can be seen that the changes between baseline and both 3 and 12 months are, in all cases, larger for the HIPC clients than they were for the HIP RCT clients (Table 4). All these differences are statistically significant (ADL $0-3$ months change $z=3.02, P=0.0025$; ADL $0-12$ months change $z=2.26, P=0.0237$; IADL $0-3$ months change $z=4.41, P<0.0001$; IADL $0-12$ months change $t=2.45, d f=101, P=0.0159$; AQOL $0-3$ months change $t=2.83, d f=143, P=0.0053$; AQOL $0-12$ months change $t=3.81, d f=143, P=0.0002$; MFES $0-3$ months change $t=-3.58, d f=92, P=0.0006$; MFES $0-12$ months change $t=-3.81, d f=141, P=0.0002$; TUG $0-3$ months change $z=2.97, P=0.0030$; TUG $0-12$ months change $t=4.03$, $d f=105, P=0.0001$ ).

Table 5 presents the results of logistic regression models and displays the effect of receiving HIPC (as compared to HIP) on the odds of recording an improvement in each outcome, from baseline to each follow-up point. In all cases, odds ratios were greater than one, indicating that receipt of HIPC was associated with an increase in the odds of recording an improvement in outcomes; however, these odds ratios were only significant in terms of the change in TUG times (baseline to 3 months 3.24 [95\% confidence interval $\{\mathrm{CI}\}: 1.67-6.28$ ], baseline to 12 months 2.19 [95\% CI: 1.27-3.77]). Additionally, in each case, worse baseline scores were associated with an increase in the odds of recording an improvement to each follow-up point (results not displayed).

\section{Service outcomes}

Table 6 presents the service outcomes for the two groups at 3 and 12 months. Overall, the outcomes for the HIPC group at 3 months appear (statistical testing of the differences was not possible due to some cells having counts below 5) to be somewhat better, with fewer deaths, hospitalizations, and transfers to residential care or to hospice; and more clients no longer requiring home care service (as opposed to recording an increase or a continuation of the same level of service). A similar pattern can also be seen at 12 months, the exception being a higher proportion in hospital in the HIPC group.

However, a slightly different picture is presented when we look only at those individuals remaining well and living in the community (Table 7). This table shows that in the HIPC group, a smaller proportion of clients, compared to the HIP $\mathrm{RCT}$, reported the poorest outcome of requiring an increase in services (27.9\% compared to $36.8 \%)$, and a greater

Table 4 Changes in outcome measure scores over time

\begin{tabular}{|c|c|c|c|c|}
\hline \multirow[t]{3}{*}{ Measure } & \multirow{2}{*}{\multicolumn{2}{|c|}{$\begin{array}{l}\text { Mean change }(n) \\
\text { Baseline to } 3 \text { months }\end{array}$}} & \multirow{2}{*}{\multicolumn{2}{|c|}{$\begin{array}{l}\text { Mean change }(n) \\
\text { Baseline to I year }\end{array}$}} \\
\hline & & & & \\
\hline & HIPC & HIP RCT & HIPC & HIP RCT \\
\hline ADL total & $-1.64(58)$ & $-0.51(88)$ & $-1.22(58)$ & $-0.31(88)$ \\
\hline IADL total & $-5.00(58)$ & $-1.69(88)$ & $-3.97(58)$ & $-1.85(88)$ \\
\hline AQOL score & $-3.86(58)$ & $-1.54(87)$ & $-4.31(58)$ & $-1.37(87)$ \\
\hline MFES score & $\mathrm{I} .40(56)$ & $0.27(87)$ & $\mathrm{I} .42(56)$ & $0.22(87)$ \\
\hline TUG time in seconds & $-8.89(44)$ & $-3.91(63)$ & $-10.03(44)$ & $-3.08(63)$ \\
\hline
\end{tabular}

Note: Numbers vary as only clients with scores at all three measurement points included.

Abbreviations: ADL, Activities of Daily Living; IADL, Instrumental Activities of Daily Living; AQOL, Assessment of Quality of Life; MFES, Modified Falls Efficacy Scale; TUG, Timed UP and Go; HIPC, Home Independence Program Coordinator; HIP RCT, Home Independence Program randomized controlled trial. 
Table 5 Logistic regression models comparing odds of there being an improvement with HIPC as compared to HIP on each outcome

\begin{tabular}{|c|c|c|c|c|}
\hline Outcome & Odds ratio & Lower $95 \% \mathrm{Cl}$ & Upper $95 \% \mathrm{Cl}$ & $P$-value \\
\hline \multicolumn{5}{|l|}{$A D L$} \\
\hline At 3 months & 1.45 & 0.97 & 2.18 & 0.072 \\
\hline At 12 months & 1.34 & 0.91 & 1.97 & 0.140 \\
\hline \multicolumn{5}{|l|}{ IADL } \\
\hline At 3 months & 1.34 & 0.90 & 2.00 & 0.156 \\
\hline At 12 months & 1.08 & 0.72 & 1.61 & $0.7 \mid 4$ \\
\hline \multicolumn{5}{|l|}{ AQOL } \\
\hline At 3 months & 1.22 & 0.82 & 1.82 & 0.316 \\
\hline At 12 months & 1.37 & 0.90 & 2.08 & 0.142 \\
\hline \multicolumn{5}{|l|}{ MFES } \\
\hline At 3 months & 1.14 & 0.76 & 1.71 & 0.536 \\
\hline At 12 months & 1.26 & 0.83 & 1.90 & $0.27 \mid$ \\
\hline \multicolumn{5}{|l|}{ TUG } \\
\hline At 3 months & 3.24 & 1.67 & 6.28 & $<0.001$ \\
\hline At 12 months & 2.19 & 1.27 & 3.77 & 0.005 \\
\hline
\end{tabular}

Note: Additional covariates of age, sex, living arrangement, and baseline score on each measure.

Abbreviations: HIPC, Home Independence Program Coordinator; HIP, Home Independence Program; Cl, confidence interval; ADL, Activities of Daily Living; IADL, Instrumental Activities of Daily Living; AQOL, Assessment of Quality of Life; MFES, Modified Falls Efficacy Scale; TUG, Timed UP and Go.

proportion reported the most positive outcome of having no need for ongoing service (52.9\% compared to $44.7 \%$ ); however, these differences were not significant $\left(\chi^{2}=1.99\right.$, $P=0.369$ ). Similarly, at 12 months, a smaller proportion of HIPC clients recorded an increase in service $(12.7 \%$ compared to $23.0 \%$ ), and a greater proportion had no need for ongoing service ( $73 \%$ compared to $58.9 \%)$; again these differences were not significant $\left(\chi^{2}=4.47, P=0.107\right)$.

\section{Discussion}

The results of the outcome evaluation of HIPC were positive. Both short-term and sustained gains were seen on all personal outcome measures, and these were translated into a reduced need for home care, with just over half of the clients at 3 months and nearly three quarters at 12 months not using any home care service at all. A further $19 \%$ and $14 \%$ were using the same or a lower level of service at these time points than at baseline, despite having been referred because their needs had increased.

When the personal outcomes results are compared with the results from the HIP RCT, they appear as good as, if not better than, when HIP was delivered by health professionals. However, as discussed in detail in the "Limitations" section, methodological difficulties in the HIP RCT mean that the personal outcomes results are not strictly comparable as baseline data were collected at slightly different time points in the two studies.

The only UK study to look at personal as well as service outcomes in relation to reablement services ${ }^{13}$ found that individuals who had received reablement as compared to usual home care scored better on all dimensions of health and social care-related quality of life measures, but the difference was largest in relation to ability to engage in everyday activities. It is not possible, however, to compare

Table 6 Service outcomes at 3 and 12 months for all HIPC and HIP RCT clients, n (\%)

\begin{tabular}{|c|c|c|c|c|}
\hline \multirow[t]{2}{*}{ Service outcome } & \multicolumn{2}{|l|}{3 months } & \multicolumn{2}{|l|}{12 months } \\
\hline & HIPC & HIP RCT & HIPC & HIP RCT \\
\hline Died & I (I.43\%) & 15 (4.87\%) & $2(2.86 \%)$ & $61(19.68 \%)$ \\
\hline Admitted to hospital & I (I.43\%) & 21 (6.77\%) & $4(5.7 \mid \%)$ & $12(3.87 \%)$ \\
\hline Residential care & $0(0 \%)$ & $16(5.16 \%)$ & I (I.43\%) & 25 (8.06\%) \\
\hline Hospice & $0(0 \%)$ & $5(1.61 \%)$ & $0(0 \%)$ & $3(0.97 \%)$ \\
\hline Same level & $13(18.57 \%)$ & $42(13.55 \%)$ & 9 (I2.86\%) & $36(11.61 \%)$ \\
\hline Increase in service & $19(27.14 \%)$ & $93(30.00 \%)$ & $8(11.43 \%)$ & 48 (I5.48\%) \\
\hline Decrease in service & $0(0 \%)$ & $5(1.61 \%)$ & $0(0 \%)$ & $2(0.65 \%)$ \\
\hline No longer receiving service & $36(51.43 \%)$ & II 3 (36.45\%) & 46 (65.7I\%) & I $23(39.68 \%)$ \\
\hline Total & $70(100 \%)$ & $310(100 \%)$ & 70 (100\%) & $310(100 \%)$ \\
\hline
\end{tabular}

Abbreviations: HIPC, Home Independence Program Coordinator; HIP RCT, Home Independence Program randomized controlled trial. 
Table 7 Service outcomes at 3 and 12 months, clients alive, nonpalliative, and not institutionalized, n (\%)

\begin{tabular}{|c|c|c|c|c|}
\hline \multirow[t]{2}{*}{ Service outcome } & \multicolumn{2}{|l|}{3 months } & \multicolumn{2}{|l|}{12 months } \\
\hline & HIPC & HIP RCT & HIPC & HIP RCT \\
\hline Same level as prior/decrease & $13(19.12 \%)$ & 47 (I8.58\%) & $9(14.29 \%)$ & $38(18.18 \%)$ \\
\hline Increase in service & $19(27.94 \%)$ & $93(36.76 \%)$ & $8(12.70 \%)$ & $48(22.97 \%)$ \\
\hline No longer receiving service & 36 (52.94\%) & II 3 (44.66\%) & $46(73.02 \%)$ & $123(58.85 \%)$ \\
\hline Total & 68 (100\%) & 253 (100\%) & 63 (100\%) & 209 (100\%) \\
\hline
\end{tabular}

Abbreviations: HIPC, Home Independence Program Coordinator; HIP RCT, Home Independence Program randomized controlled trial.

the degree of improvement in personal outcomes in their study with the results of our evaluation as different measures were used.

Service outcomes for the HIP RCT were not informed by data collected at client interviews and are therefore comparable with the service outcomes achieved for HIPC. These outcomes were found to be fairly similar for HIP in the two studies, although the HIPC group were less likely to have shifted to residential care at either follow-up point than clients who received HIP in the RCT. This could have been expected considering the differences in eligibility for HIP between the two studies. Among those clients alive, nonpalliative and not receiving residential or hospital care, there were no differences in the level of Silver Chain services being received at each follow-up point. As noted in the "Findings" section, receiving the same or a reduced level of services at follow-up, like not needing any services, can also be seen as indicating a positive outcome given that clients were referred because they had requested or were referred for a new service or an increase in service level. Thus, in service terms, the outcomes were positive for about two-thirds by 3 months, and over three quarters at 12 months, of clients in both studies. Although somewhat lower than the best UK research results $-61 \%$ using no services and $26 \%$ having a reduced level of service at 3 months $-{ }^{1}$ the results are at the top of the range of the results achieved by three-quarters of the reablement services looked at by Glendinning and Newbronner ${ }^{26}$ in 2008.

As regards the generalizability of this study's findings to the broader HACC population, while demographically similar in many respects to the WA and National HACC populations, our study sample did include proportionally more women, more people who lived alone, and more people born in a country other than Australia. ${ }^{27,28}$ While living alone could well act to motivate people to optimize their function to retain their independence, it is not immediately apparent why being female or being born overseas should influence success in reablement. Further research to identify the characteristics associated with positive reablement outcomes is needed.

\section{Limitations}

The timing of data collection in this evaluation was somewhat different to that in the RCT. Operational concerns that services could be delayed if baseline data were collected prior to service commencement meant that in the RCT, baseline data were sometimes not collected for more than a week after services had commenced. As a consequence, many individuals had already made functional gains before the baseline data were collected. This was demonstrated by the percentage of RCT clients able to shower themselves having increased from $9 \%$ at referral to $49 \%$ at baseline. ${ }^{2}$ Additionally, it resulted in the ADL and IADL baseline mean scores for clients in the RCT indicating lower dependency than the clients in the present evaluation. Yet, to be eligible for the RCT, it was necessary to have a PC (ADL) need, whereas individuals with only IADL needs were eligible for HIPC. Having observed the impact of delayed baseline measures in the RCT, a different process was followed in the HIPC evaluation. After negotiation with HIPC management and the coordinators themselves, a way of combining baseline data collection with the first service visit was agreed. When tried, it was found to work well for all parties concerned and means that the change due to the intervention is more accurately quantified in the HIPC evaluation than it was in the RCT, in which improvements are likely to have been underestimated. In this analysis, differences in baseline scores were accounted for by inclusion in logistic regression models. Poorer scores were found to be associated with an increased likelihood of improvement on each outcome measure; however, even when controlling for these baseline differences, HIPC clients were shown to be as likely or more likely to record an improvement in outcomes as compared to HIP clients.

Unfortunately, due to the lower-than-expected referral rate to the service, the coordinators' caseloads were lower than optimum in terms of productivity during the evaluation period. This meant that they were able to spend more time with their clients, and this was reflected both in the activity data as well as the very high level of client satisfaction with this aspect of the service. It also meant that in some cases, the 
coordinators provided types of support that may otherwise have been allocated to Home Support providers. It is not therefore possible to comment at this point in time on the potential cost-effectiveness of the service delivered by coordinators compared to when delivered by health professionals. It should also be noted that the HIPC service was delivered in the context of a multidisciplinary team who provided mentoring and support to the coordinators as well as specific expertise and the availability of specialist referral if required. It cannot be assumed that similar outcomes would be achieved by a HIP coordinator service without the support of a broader multidisciplinary team. That remains to be tested.

The lower-than-expected referral rate may also have impacted on our ability to make inferences regarding any difference in effect between the HIP and HIPC programs. Under the final sample of 70 HIPC clients, the study was powered to detect a difference in service outcomes of $16 \%$ in comparison to HIP clients. Given that $15 \%$ more HIPC clients had no need for ongoing service at 12 months follow-up, it is possible that a significant difference may have been detected had the full intended sample of 100 clients been recruited. Alternatively, it is possible that sampling error may have contributed to the difference in proportions requiring ongoing service between the groups; had 100 clients been recruited, this difference may in fact have been smaller. It appears extremely unlikely, however, that having the full sample of 100 clients could have resulted in the finding that outcomes were significantly worse in the HIPC group. Testing in which 30 fictional clients with a need for ongoing care (ie, all with a negative outcome) were added to the existing sample showed no significant difference between groups. Although the reduced sample size is problematic, it appears unlikely that this alone has led to the interpretation of results that HIPC appears to perform as well as or better than the original HIP intervention.

\section{Conclusion}

About 70 clients who received the HIPC service and participated in this evaluation made and sustained significant gains in their everyday functioning, mobility, confidence, and well-being that translated into a reduced need for home care services going forward. These results were found to be comparable with the outcomes achieved in the HIP RCT when the service was delivered by health professionals.

There is currently limited evidence concerning which elements of restorative home care/reablement service models are critical in determining their effectiveness; how such services can be most cost-effectively delivered; and how their effectiveness may depend upon the characteristics of the service recipient. These findings add evidence that such services may be effectively delivered in Australia with care managed by specifically trained non-health professionals, provided multidisciplinary support is available, and that services may be similarly effective for those with a range of ADL/IADL limitations as they are for the population with specific PC needs.

\section{Acknowledgments}

This research would not have been possible without the following: funding for the development of the HIPC service delivery model and training from Health Workforce Australia; funding for the HIPC trial and evaluation from the WA Department of Health Aged and Continuing Care Directorate; project management by Suzanne Vandermeulen; assistance from the HIPC coordinators; support of HIPC management; and the willingness of the clients to be involved.

\section{Disclosure}

The authors report no conflicts of interest in this work.

\section{References}

1. Francis J, Fisher M, Rutter D. Reablement: A Cost-Effective Route to Better Outcomes. London, UK: Social Care Institute for Excellence; 2011.

2. Lewin G, De San Miguel K, Knuiman M, et al. A randomised controlled trial of the Home Independence Program (HIP), an Australian restorative home care programme for older adults. Health Soc Care Community. 2013;21(1):69-78.

3. Lewin G, Vandermeulen S. A non-randomised controlled trial of the Home Independence Program (HIP): an Australian restorative programme for older home-care clients. Health Soc Care Community. 2010; 18(1):91-99.

4. Newbronner E, Baxter M, Chamberlain R, Maddison J, Arksey H, Glendinning C. Research into the Longer Term Impacts/Effects of Re-ablement Services, Care Services Efficiency Delivery Program. London, UK: Department of Health Physical Activity Health Improvement and Prevention; 2007.

5. King AL, Parsons M, Robinson E, Jörgensen D. Assessing the impact of a restorative home care service in New Zealand: a cluster randomised controlled trial. Health Soc Care Community. 2012;20(4):365-374.

6. Parsons M, Anderson C, Senior H, et al. ASPIRE: Assessment of Services Promoting Independence and Recovery in Elders. Auckland, New Zealand: The University of Auckland; 2007.

7. Lewin G, Allan J, Patterson C, Knuiman M, Boldy D, Hendrie D. A comparison of the home-care and healthcare service use and costs of older Australians randomised to receive a restorative or a conventional homecare service. Health Soc Care Community. 2014;22(3):328-336.

8. Tinetti M, Baker D, Gallo W, Nanda A, Charpentier P, O'Leary J. Evaluation of restorative care vs usual care for older adults receiving an acute episode of home care. J Am Med Assoc. 2002;287(16):2098-2105.

9. Tinetti M, Charpentier P, Gottschalk M, Baker D. Effect of a restorative model of posthospital home care on hospital readmissions. J Am Geriatr Soc. 2012;60(8):1521-1526.

10. Lewin G, Alfonso H, Alan J. Evidence for the long term cost effectiveness of home care reablement programs. Clin Interv Aging. 2013; $8: 1273-1281$ 
11. Cochrane A, McGilloway S, Furlong M, Molloy DW, Stevenson M, Donnelly M. Home-care 're-ablement' services for maintaining and improving older adults' functional independence. Cochrane Database of Systematic Reviews 2013, Issue 11; Art No CD010825.

12. Ryburn B, Wells Y, Foreman P. Enabling independence: restorative approaches to home care provision for frail older adults. Health Soc Care Community. 2009;17(3):225-234.

13. Glendinning C, Jones K, Baxter K, et al. Home Care Re-ablement Services: Investigating the Longer-Term Impacts (Prospective Longitudinal Study). New York, NY: Social Policy Research Unit, University of York; 2010.

14. Parsons J, Sheridan N, Rouse P, Robinson E, Connolly M. A randomised controlled trial to determine the effect of a model of restorative home care on physical function and social support among older people. Arch Phys Med Rehabil. 2013;94:1015-1022.

15. Lewin G, De San Miguel K, Knuiman M, et al. A randomised controlled trial of the Home Independence Program, an Australian restorative home-care programme for older adults. Health Soc Care Community. 2013;21(1):69-78

16. Care Services Efficiency Delivery Programme. Homecare Re-ablement Workstream: Discussion Document. London, UK: Department of Health; 2007.

17. Social Care Institute for Excellence. Reablement: A Key Role for Occupational Therapists. London, UK: Social Care Institute for Excellence; 2011.

18. Lewin G, Calver J, McCormack B, et al. The Home Independence Project. Geriaction. 2008;26(3):13-20.
19. Colin C, Wade D, Davies S, Horne V. The Barthel ADL index: a reliability study. Int Disabil Stud. 1988;10:61-63.

20. Lawton M, Brody E. Assessment of older people: self-maintaining and instrumental activities of daily living. Gerontologist. 1969;9: 179-196.

21. Calver J, Lewin G, Holman C. Reliability of a primary generic assessment instrument for home care. Australasian J Ageing. 2002;21: 185-191.

22. Hawthorne G, Richardson J, Osborne R, McNeil H. The Australian Quality of Life (AQoL) Instrument: Initial Validation. West Heidelberg, VIC: Centre for Health Program and Evaluation; 1997.

23. Hill K, Schwarz J, Kalogeropoulos A, Gibson SJ. Fear of falling revisited. Arch Phys Med Rehabil. 1996;77(10):1025-1029.

24. Podsiadlo D, Richardson S. The timed up and go: a test of basic functional mobility for frail elderly persons. J Am Geriatr Soc. 1991;39: 142-148.

25. Statacorp. Stata Statistical Software: Release 13. College station, TX: Statacorp LP; 2013.

26. Glendinning C, Newbronner L. The effectiveness of home care reablement - developing the evidence base. J Integr Care. 2008;16(4): 32-39.

27. Home and Community Care (HACC). Home and Community Care Program Minimum Data Set 2013-2014 Annual Bulletin. Canberra, ACT: Australian Government Department of Social Services; 2015.

28. Home and Community Care (HACC) Program. Minimum Data Set Report 2013-2014 Annual Report. Canberra, ACT: Australian Government Department of Social Services; 2014.
Clinical Interventions in Aging

\section{Publish your work in this journal}

Clinical Interventions in Aging is an international, peer-reviewed journal focusing on evidence-based reports on the value or lack thereof of treatments intended to prevent or delay the onset of maladaptive correlates of aging in human beings. This journal is indexed on PubMed Central, MedLine,

\section{Dovepress}

CAS, Scopus and the Elsevier Bibliographic databases. The manuscript management system is completely online and includes a very quick and fair peer-review system, which is all easy to use. Visit http://www.dovepress. com/testimonials.php to read real quotes from published authors. 